\title{
Metrological study of CFRP drilled holes with x-ray computed tomography
}

\author{
Nadia Kourra • Jason M. Warnett • Alex Attridge • \\ Ercihan Kiraci • Aniruddha Gupta · Stuart Barnes • \\ Mark A. WIlliams
}

Received: 4 September 2014 / Accepted: 17 December 2014 / Published online: 22 January 2015

(c) Springer-Verlag London 2015

\begin{abstract}
The popularity of composite materials is continuously growing with new varieties being developed and tested with different machining processes to establish their suitability. Destructive as well as non-destructive methods, such as ultrasonics, X-ray radiography and eddy-current, have previously been used to ensure that the combination of particular machining methods and composites provide the required quality that can allow the required lifespan of the final product. X-ray computed tomography (CT) is applied as a novel method in this paper to obtain quantitative data about the inner and outer structures of carbon fibre reinforced polymer (CFRP) drilled holes providing more information than any other non-contact and non-destructive evaluation. This is combined with precise measurements from optical CMM and image processing for a full analysis for the entire part. This method can provide accurate measurements for all the layers of the CFRP and very little interaction from the operator minimising the human error. The method complies with VDI/VDE 2630 standard and the quality of the acquired measurements is assured. The results can assist in establishing the best machining process, provide accurate measurements of diameter, circularity and positioning of the hole and information about delaminated areas.
\end{abstract}

Keywords Metrology · Computed tomography - CFRP . Drilling $\cdot$ Non-destructive evaluation $\cdot$ Non-contact inspection

N. Kourra $(\bowtie)$ - J. M. Warnett - A. Attridge - E. Kiraci ·

A. Gupta $\cdot$ S. Barnes - M. A. WIlliams

WMG, The University of Warwick,

Coventry CV4 7AL, UK

e-mail: N.Kourra@warwick.ac.uk

\section{Introduction}

In the recent years, there is a growing necessity to reduce the weight and increase the strength of structures produced in aerospace and automotive industry. As a result, there is an increasing interest in composite materials in these industries. Composite materials have been developed over the last two decades when materials with specific properties are required. They are formed by combining two or more distinct phases of more than one material using a reinforcement material to the matrix material, enhancing the properties of each distinct phase for particular applications [1-4].

The applications of carbon fibre reinforced polymers (CFRPs) in aerospace and automotive industries are well known, such as fan cases, vanes and fan blades [3]. The mechanical and structural properties of CFRPs depend on the nature, orientation and bond of the fibres that are arranged in flat panels, sheets or a woven structure. The popularity of CFRPs in these industries is due to their advanced mechanical properties such as low-specific weight, strength to weight ratio, durability, stiffness and density. These characteristics permit higher specific strength and stiffness in light structures while their high cost limits its applications to cases when performance is the most important consideration. The greatest limitations of CFRPs are their heterogeneity and heat sensitivity. They are also anisotropic and their material behaviour depends on diverse reinforcement and the matrix properties [1,2, 4-10].

The machining of CFRPs is affected by these limitations and it is often required to determine the most suitable machining method for particular application. In this paper, a new method has been developed to study the defects of CFRPs caused by different drilling procedures, assisting in the identification of the superior one. This method utilises X-ray computed tomography (CT) and image 
processing, providing data of the outer surfaces of the part, the surface of the hole and the laminated layers of the composite.

\section{Background}

The utilisation of composites is growing and their design is getting more complicated as the requirements increase. Machining of composites and especially CFRPs is affected by the nature of the composites and it result to affect the properties of the finished parts. Destructive and nondestructive methods have been applied to better understand the way that different factors affect the service life of a part. Improvements to machining processes are constantly researched, being developed and evaluated. CT is such a method that can be used to examine and evaluate the results of machining processes non-destructively, providing data for the entire part.

\subsection{Machining carbon fibre reinforced polymers (CFRP)}

The selection of machining processes and tools has to be done according to the properties of the materials, tool wear mechanisms and the requirements of the application. The behaviour of the matrix material differs from the response of the reinforcement material while they are machined and both behaviours have to be considered before the selection of the machining process and tool.

Due to the difficulties faced when composites materials are machined, near-net shape processes are often applied to avoid any damage that would adversely affect their strength $[8,9]$. However, some machining processes are unavoidable since mechanical fasteners are often required. The most common secondary machining applied in CFRPs is drilling, which may cause fragmentation, burrs, interlaminar cracks, interfacial de-bounding, thermal damages and fibre pull-out, all of which can lead to delamination, as indicated in Fig. 1 $[2,4,8,10,11]$.

Several studies, both analytical and experimental, look into the different factors that influence the quality and performance of CFRPs and demonstrate how delamination is affected by tool thrust and torque. The thrust force and torque continually increases while cutting the layers and the maximum thrust force and torque are achieved when the bottom layer is reached [2]. Delamination can be minimised by varying feed rate to control the thrust force [7, 12]. The effects of critical thrust force can be controlled by the use of backup plate [9]. Cutting speed, feed rate and point angle of the drill affect delamination and excessive thrust force and torque can generate heat through friction and cause matrix damage such as softening of the matrix [13-15].
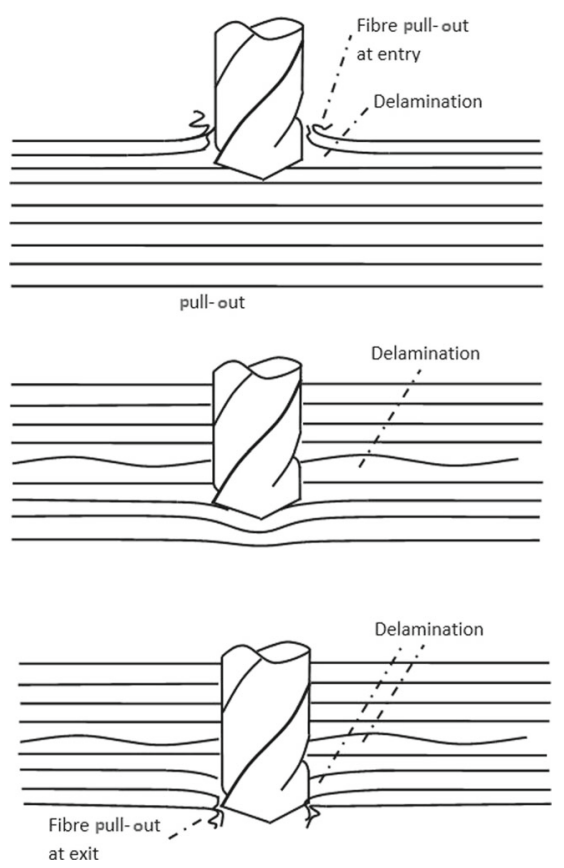

Fig. 1 Machining may cause de-bounding, fibre pull out and delamination

The tool geometry, material and type have also been largely researched $[5,16,17]$. The different characteristics of a drill such as the cutting angle, cutting edge and the tip angle affect the drilling of composite laminates [5]. Researchers suggested new types of drills with specific geometries to minimise defects and delamination [18, 19]. Investigations of drill material, shape and size also took place to determine the factors that affect the formation of delamination $[7,9,20]$.

It can be concluded from the research in this field that the design, geometry and material of the drill can affect the result of the machining process. New methods and tool designs are continually under development with ultrasonic assisted drilling being one of them [21].

\subsection{Non-destructive testing of CFRPs}

Due to these numerous factors that affect the quality of drilled holes in CFRPs, several techniques were previously employed to assess the different results. Destructive methods can be applied by cutting the part of drilled hole to be able to examine it, but as a result part of the hole is destroyed and some potentially useful data is lost. For this reason, non-destructive test techniques are favoured to identify and study damage, defects and faults caused by the machining processes. Optical inspection with microscope is commonly used by researchers who consider machining experiments, for example, Davim and Reis [7]. The most common techniques applied that provide more data than from the surface 
have been radiography, ultrasonics and eddy-current testing. De Goeje and Wapenaar [22] illustrated that eddy-current examinations can establish the type of defects and can show the orientation of fibres. Persson et al. [5] used 2D X-ray techniques to detect and analyse the extent of defects in their investigation.

Several researchers introduce more unconventional techniques to reduce the testing time, cost and improve the quality of the collected data. Pye and Adams [23] discussed the use of infra-red thermography to study the variations of surface temperature while the structure is under load, demonstrating that the combination of resonant vibration and thermography can detect damage in composite materials. Further, Mook et al. [24] used special probes that emit eddy-currents to visualise fibre orientation, local imperfections such as fibre fractions, resin rich zones, delamination and impact damages. Mourtiz et al. [25] investigated whether pulse echo ultrasonics can detect the formation and growth of fatigue damage in thick GRPs and CFRPs. Steinberger et al. [26] demonstrated infrared thermographic technique to characterise defects in CFRPs during fatigue tests that use a photothermal heat source and the heat conductivity was used to determine the damage in the samples. Davim et al. [27] used digital image analysis to overcome the limitations of visual inspection in their analysis to determine delamination factors in drilled CFRPs. Seif et al. [28] used a shadow moire laser based on an imaging technique and demonstrated the possibility of determining the extent of hidden delamination. According to the authors, the developed technique provides accuracy and simplicity. Raisutis et al. [29] showed the application of guided waves which inspect small diameter CFRP rods.

Based on the results of these studies, all the applications of non-destructive tests in the field have their limitations and are chosen according to the requirements of the investigation. The most important limitations of the techniques used in the past are their limited data with constrained accuracy and their subjectivity to human error. The obtained data from these techniques is limited to a small number of measurements, and they are unable to study the entire surface of the hole, concentrating instead on the entrance and exit surfaces and layers within close proximity of these regions. It can be concluded that a non-contact and non-destructive evaluation with high accuracy that can provide data for the entire specimen, without being susceptible to human error, is required.

\subsection{X-ray computed tomography}

X-ray Computed Tomography (CT) uses X-rays to collect radiographs that are reconstructed into a $3 \mathrm{D}$ model. The reconstruction data includes the inner structure of the scanned part as well as the outer surface.
In an X-ray radiograph each pixel has a grey value that represents the attenuation of the X-ray after it penetrates the scanned object. Numerous 2D radiographic projections are collected during a CT scan through $360^{\circ}$ rotation of the object and they are then reconstructed with Filtered Back Project (FBP). These algorithms digitally rebuild the part. When the radiographs are reconstructed the grey values of each pixel are converted to represent the grey values of a corresponding volume called voxel, as shown in Fig. 2. This $3 \mathrm{D}$ volume can then be exported for analysis [30-33].

CT has previously been shown to provide useful data for examinations of CFRP and is capable to assess entrance and exit delamination of drilled holes [9]. A more recent study by Perjyd et al. [3] utilised CT to examine surface integrity of CFRP drilled holes to assist with the determination of the optimum machining procedures. In this study, it is demonstrated that the complete inner hole surface and near surface region can be inspected. CT can also provide information on all the laminates throughout the hole that may have been affected by the drilling with a focus on the quality of finished parts, particularly relevant in aerospace industry.

This technology can be applied in non-destructive evaluations and as a metrological tool when strict procedures are followed to overcome a series of issues that can affect the precision of the data. The collected CT data has been proven to be possibly influenced by bi-directional repeatability of the system, radial run-out errors, the detector tilt and type and the movement of the spot size of the X-ray in the duration of a scan [34-38]. The reduction of artefacts in affected data can be achieved by following the recommendations provided by VDI/VDE 2630 Part 1.4; a guide to obtain dimensional measurements through CT [39].

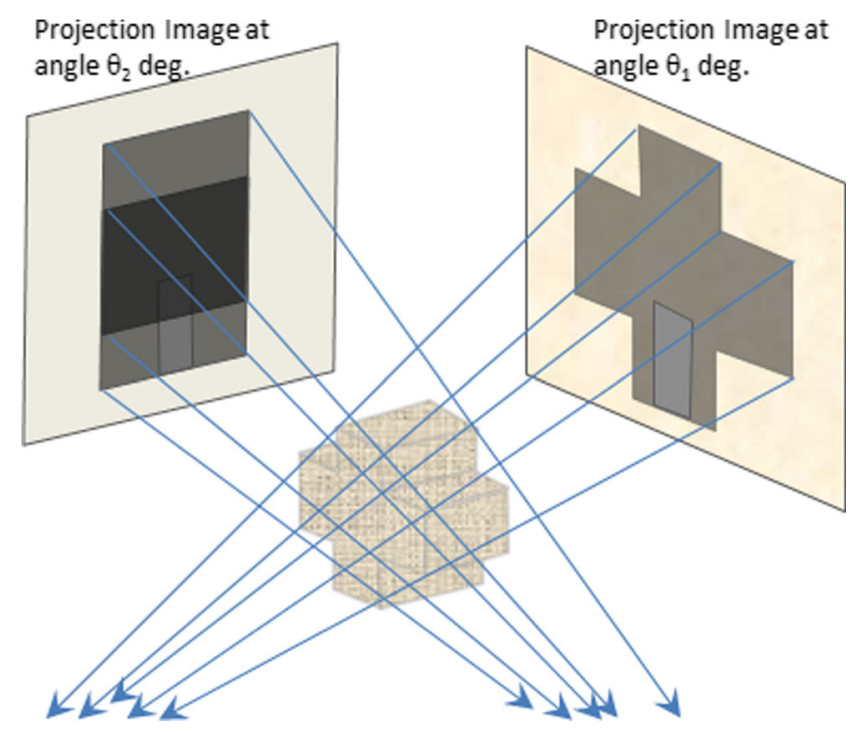

Fig. 2 FBP rebuilds a 3D model of the scanned parts with voxels 
There are four different measuring procedures that can be used in CT scanning, ultimately leading to dimensional metrological results. One of the applicable methods describes how to make nominal/actual comparison between the scanned data and a reference measurement. The CT measurements may be subject to absolute scale errors that correspond to the wrong voxel size. According to Lifton et al. [40], the measurement error in CT is mainly systematic. A compensation can be made by comparing and scaling the CT results with reference measurements. The results are typically affected by less than $1 \%$ error which can be reduced with this method to less than $0.2 \%$ $[39,40]$.

\section{Methodology}

There are several factors that affect the quality of drilled holes, established by the accepted tolerances and the delamination caused during the machining. An examination is required that can provide data on the size and location of the defects and any potential delamination. X-ray CT can locate the defects and characterise them as critical or not by image processing [41, 42].

The method discussed in this paper was developed to evaluate the quality of the holes with two different drilling techniques used to prove its effectiveness. The two drilling procedures are ultrasonic-assisted drilling (UAD) and conventional drilling (CD). For each technique, four holes have been examined to prove the effectiveness of each drilling process. The machining of the holes is not discussed here, but can be found in a previous paper [21]. The evaluation will be made by studying the exit delamination, internal damage, circularity and centre deviation. The method was separated in three parts: the preparation of the parts, the scanning and measurement method and the image processing.

\subsection{Preparation of the parts}

All holes were machined on woven carbon fibre composite with bismaleimide (BMI) matrix. The machine used for the preparation of the parts was ULTRASONIC 65 mono BLOCK $^{\circledR}$ (DMJ Mori Seiki, Germany) machine with an ultrasonic actuator built into the tool holder. The drilling took place with an uncoated tungsten carbide drill that has three flute twist. The characteristics of the drill can be found in Table 1. This particular drill was selected to achieve the best possible accuracy and surface finish. The drilling was performed under dry-conditions and it was achieved with the utilization of reverse piezoelectric effect.
Table 1 Characteristics of the drill

\begin{tabular}{ll}
\hline Characteristic & Value \\
\hline Diameter (mm) & 6 \\
Overall length (mm) & 66 \\
Point angle $\left(^{\circ}\right)$ & 150 \\
Rake angle & Flat angle \\
\hline
\end{tabular}

Four holes were produced with UAD, the frequency of the vibrations was $37.22 \mathrm{kHz}$ and the maximum oscillation amplitude was $2.9 \mu \mathrm{m}$. The settings were automatically selected by the machine, according to the tool and the tool holder.

The location of each hole was marked prior to the drilling by four slots as shown in Fig. 3 to identify the indented centre of the hole, $c_{\text {ideal }}$, with the positioning accuracy of the holes and slots $<8 \mu \mathrm{m}$. Both UAD and CD used the same cutting speed of $0.0157 \mathrm{~m} / \mathrm{s}$. All of the slots were produced by a $2-\mathrm{mm}$ diameter, Q-coat tungsten carbide slot drill with 2-flute twist, with cutting speed of $18,833 \mathrm{~m} / \mathrm{s}$, cross feed $0.042 \mathrm{~m} / \mathrm{s}$ and depth feed $0.0017 \mathrm{~m} / \mathrm{s}$. They have thickness of $2 \mathrm{~mm}$, depth of $0.7 \mathrm{~mm}$ and length $8 \mathrm{~mm}$.

\section{2 $\mathrm{CT}$ acquisition and initial processing}

$\mathrm{X}$-ray CT is affected by a number of variables as discussed in Section 2.3. In this phase, the best possible acquisition of the data is achieved by selecting the optimum settings

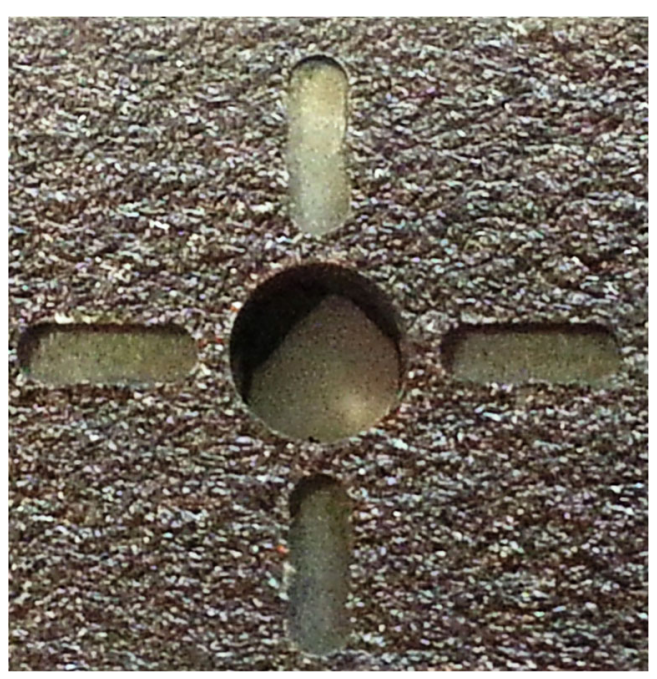

Fig. 3 The slots are perpendicular to one another, with their bisectors meeting at the intended centre, $c_{\text {ideal }}$ 
Table 2 Selected CT settings

\begin{tabular}{ll}
\hline CT settings & \\
\hline Voltage $(\mathrm{kV})$ & 215 \\
Power $(\mathrm{W})$ & 6 \\
Exposure time $(\mathrm{s})$ & 4 \\
Gain $(\mathrm{dB})$ & 18 \\
Voxel size $(\mu \mathrm{m})$ & 15 \\
\hline
\end{tabular}

as shown in Table 2 and positioning the part for maximum magnification and reduction of potential artefacts. Each part was CT scanned, Fig. 4, the acquired data reconstructed, reviewed in inspection software and then analysed with image processing. In addition, the parts were measured on a CMM to later be used for voxel scaling. The scanners and software used can be found in Table 3 .

The reconstructed data is represented by different grey values. The selection of a threshold in an inspection software separates the material from the surroundings, therefore, the selection of a threshold affects greatly the measurements collected from CT. In order to overcome this threshold limitation, the samples were measured with an optical CMM scanner just after being scanned to gain a threshold independent measurement and the voxel size was recalculated accordingly as per Lifton et al. [40].

The threshold independent measurements obtained for this analysis were the distances between the top and the lower cylindrical centres of the slots as shown in Fig. 5. The procedure followed for the voxel rescaling corresponds

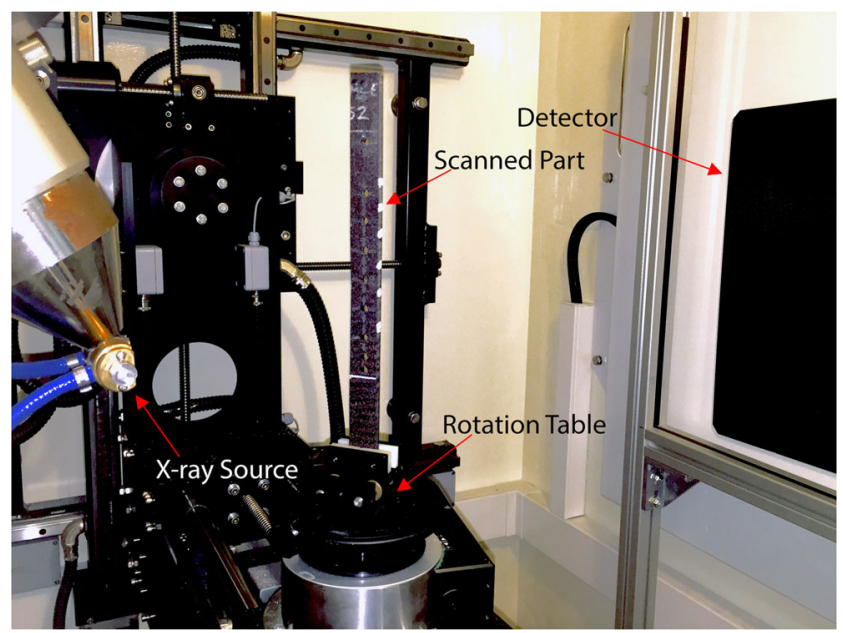

Fig. 4 Experimental set-up inside X-ray CT scanner to VDI/VDE 2630 Part 1.4. Moreover, the accuracy of the measurements is improved by rescaling the voxel size, according to research. The error of the measurements is often less than $0.2 \%$ after voxel corrections $[39,40]$.

Then, the 3D model was aligned parallel to $y-z$ plane according to the slots around the hole and then exported as DICOM images for further analysis. The number of the images is determined by the number of voxels that construct the 3D model, approximately 450 images in this instance.

\subsection{Image processing}

The image processing was performed in MATLAB with DICOM images exported from VG Studio Max 2.2. Initially, a slice is selected manually close to the entrance surface of the hole that includes the representation of the slots. The image is transformed to a binary image with the threshold selected by the Otsu method [43]. The slots are used to identify the centre of the circle, where the intersection of their bisectors represent the ideal centre, $c_{\text {ideal }}$. Imaging processing then utilises Hough transform to identify lines with slope of $0^{\circ}$ and $90^{\circ}$ with two lines identified for each slot. The mid-point between the two edges of a horizontal slot $\left(0^{\circ}\right)$ gives the $y$-coordinates of the centre of the circle while the mid-point of a vertical slot $\left(90^{\circ}\right)$ gives the $x$-coordinates as shown in Fig. 6 .

Next, the code is run continuously through all the DICOM images to calculate the maximum and minimum radius, maximum and minimum diameter, circularity, positioning and the delamination close to the exit surface, as shown in Fig. 7. The important factors related to the quality of the diameter and positioning are easily measured by traditional means while circularity of the hole is often measured distance amplifying instruments (DTI) [44].

Radii are calculated as the distance between the ideal centre $c_{\text {ideal }}$ identified by the slots and all the edges of the circle, identified using the Canny method. The maximum and minimum radii are saved separately for each image. The radii are interpolated for 1440 fixed angular quantities, for equivalent comparison across all images. The diameter differs from the radius due to the non-linearity of defects and depends on the particular edge point from which is calculated. Diameters are the measurement taken in production since radius is impossible to be measured. The diameter is calculated as the distance between the two points that intersect the circle edge and a line of prescribed gradient that passes through $c_{\text {ideal }}$.

The circularity of the hole is calculated according to ASME Y 14.5M 1994 that states: circularity is a 
Table 3 Machines and software used in this method

\begin{tabular}{llll}
\hline Machines and software used & Name & Producer \\
\hline X-ray CT scanner & X-TEK XT H 225/320 LC & Nikon Metrology, UK \\
Optical CMM scanner & NEXIV VM2 - R6555 & Nikon Metrology, UK \\
CT Reconstruction software & CT Pro 2.3 & Nikon Metrology, UK & Volume Graphics GmbH, Germany \\
CT Inspection software & VG Studio Max 2.2 & Visualization Science Group, FEI, USA \\
CT Inspection software & Avizo ${ }^{\circledR}$ Fire & MathWorks, USA & 2014 \\
Analysis Software & Matlab & & 2014 \\
\hline
\end{tabular}

condition where all the points of a surface of revolution at any section are perpendicular to a common axis [44]. In this particular case, the circularity is calculated as the difference between the maximum and minimum radius.

The code identifies the represented circle in the picture, and its $c_{\text {actual }}$ by circular Hough transform. The positioning is calculated as the absolute value of the difference between the actual and ideal centre, $\left|c_{\text {actual }}-c_{\text {ideal }}\right|$. The delaminated area is calculated by the sum of pixels that do not represent material outside the circle but they are connected with the circle.

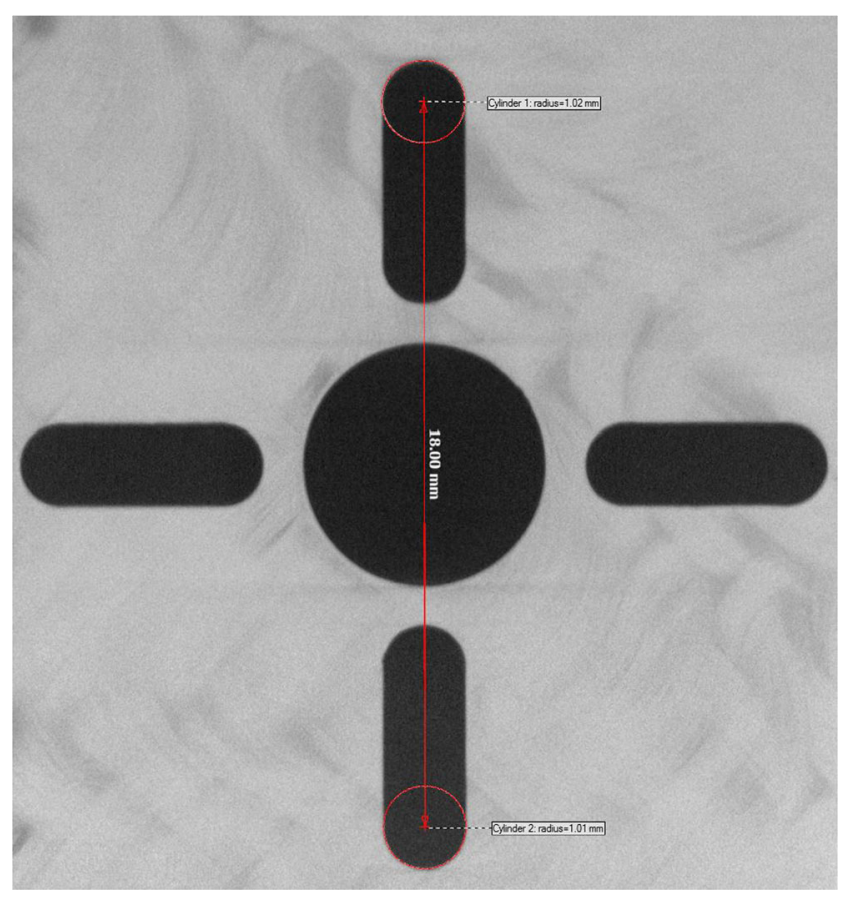

Fig. 5 Voxel rescaling according to CMM measurements. This centre to centre distance is scaled to the actual centre to centre distance measured by optical CMM

\section{Results}

\subsection{Morphological observations}

Initially, the morphology of the hole was considered to gain a general understanding of the defects. Figure 8 provides the reverse surface of the hole negating entry and exit layers with all the defects clearly shown. Protrusions in this view represent the defects, making it easier to qualitatively assess the surface of the hole.

The CT data were then examined as $2 \mathrm{D}$ images to identify the problematic areas, as shown in Fig. 9. Here, a 2D image of the cross section of the hole demonstrates that drilling can cause delamination that is not connected to the walls of the hole in any plane. This issue has not been considered in this study and further analysis can be performed to quantify this delaminated area.

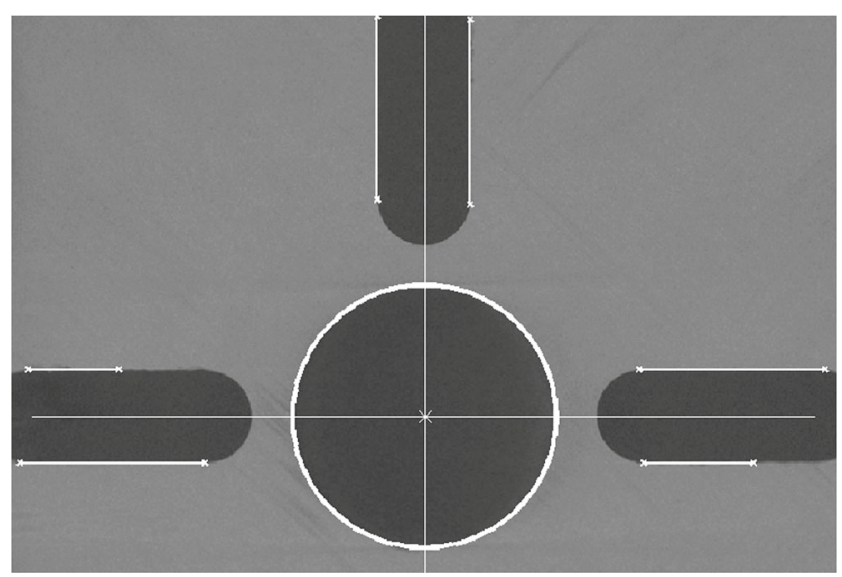

Fig. 6 Identification of the $c_{\text {ideal }}$ based on the slots. Lines of slots found by the Hough transform, and the circle periphery is found by the Cunny method 


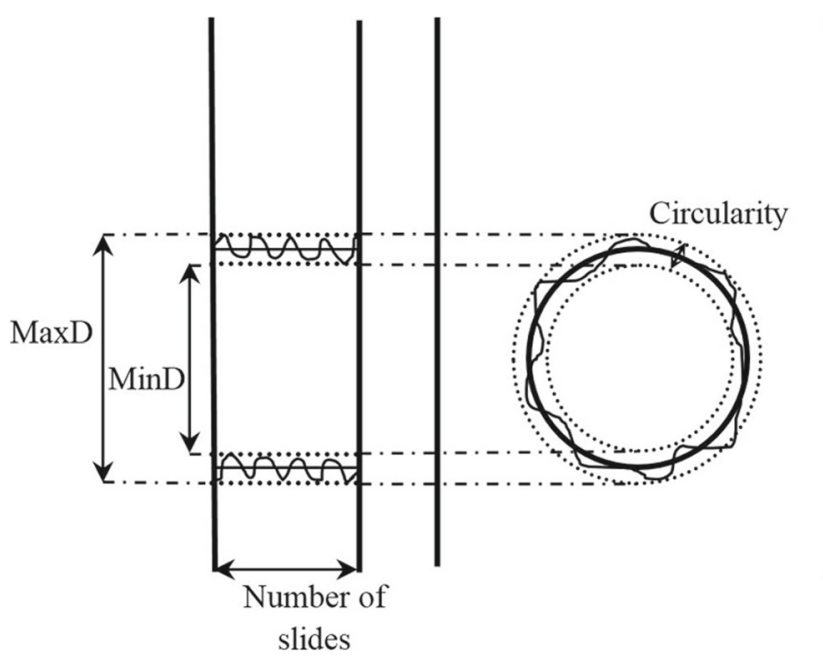

Fig. 7 Identifying the maximum and minimum diameter and calculating the circularity for each slide

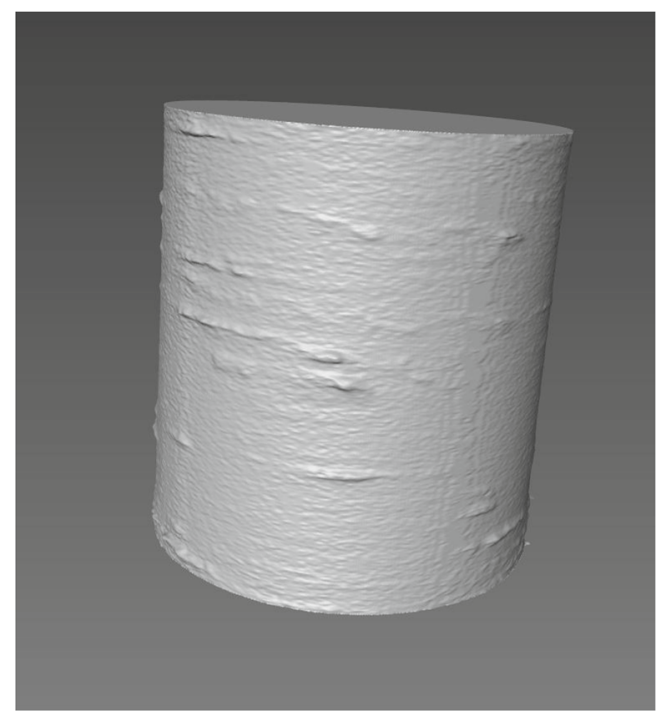

Fig. 8 The reverse surface of the hole demonstrates the inclusions as protrusions
Table 4 Expected tolerances when drilling CFRPs

\begin{tabular}{lll}
\hline Tolerances type & Symbol & Tolerances values(mm) \\
\hline Diameter & $\varnothing$ & $6+0.075$ \\
Circularity & $\bigcirc$ & $0+0.200$ \\
Positioning & $\varnothing$ & $0 \pm 0.075$ \\
\hline
\end{tabular}

\subsection{Quantitative results}

All of the quantitative results were calculated based on the required centre of the holes, $c_{\text {ideal }}$, that were identified by the slots that were produced prior to the drilling. Calculation of radii has not previously been possible on a slice by slice basis, leading to a more meticulous evaluation of quality. The analysis of the results can provide information about every defect on the surface of the hole. However, not all defects are critical and the tolerances of acceptable values of the variables are provided in Table 4.

A CD hole was digitally unravelled in Fig. 10 from a cylinder to a plane, providing a non-planar view. Here, the radius from $c_{\text {ideal }}$ is shown through 1440 discrete angular values throughout the length of the hole. The variation is immediately evident, with problematic areas readily identifiable. This figure indicates a positioning misalignment with the radii close to $150^{\circ}$ to be less than $2.95 \mathrm{~mm}$ and the radii close to $330^{\circ}$ to exceed $3.05 \mathrm{~mm}$. Another remarkable observation is the perpendicular to the drilling areas that are affected by delamination. They are approximately 20 problematic areas that exceed $3.05 \mathrm{~mm}$. This indicates that particular areas of the material are weaker than others. Values that are represented in dark red indicate delamination. The degree of delamination is worse at the exit and interestingly varies through $360^{\circ}$. The magnitude of delamination at the exit of the hole can be examined further in Fig. 11.
Fig. 9 Delamination can be detected that is not connected to the wall but can still affect the quality of the part

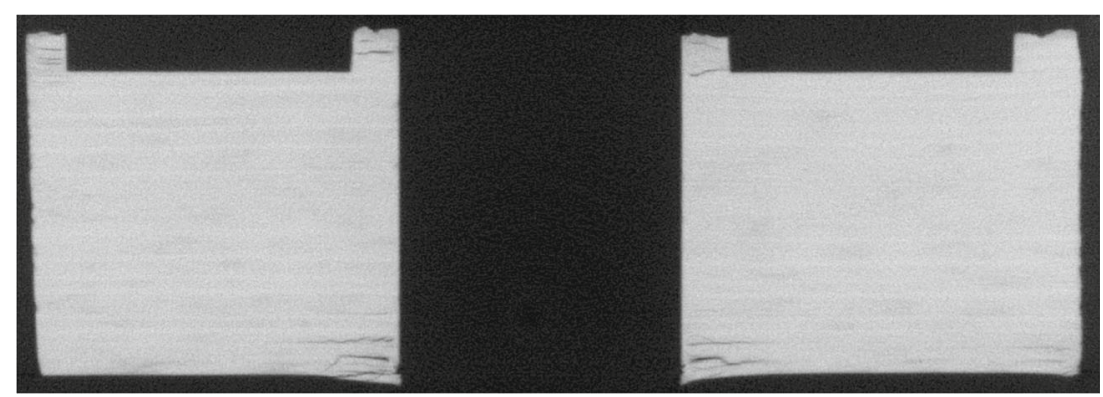




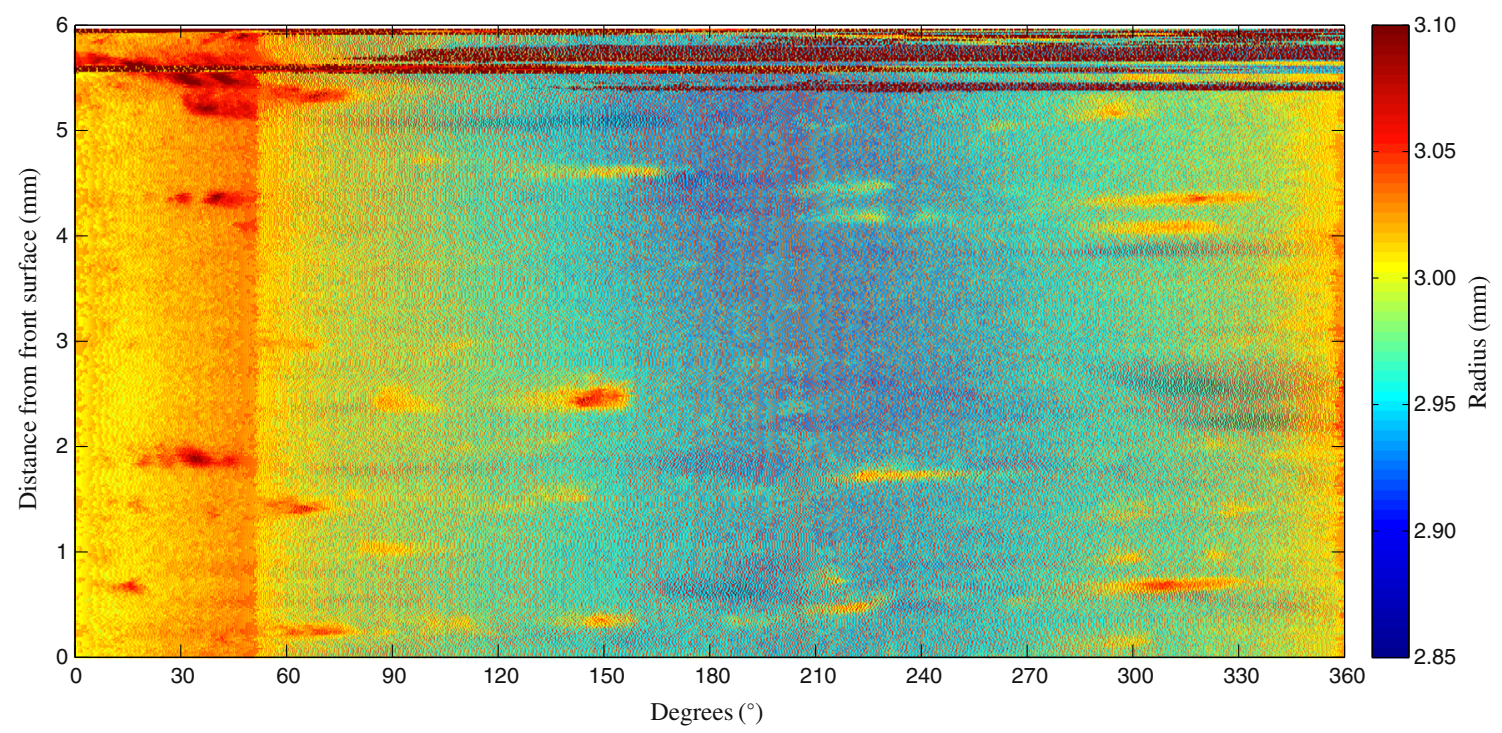

Fig. 10 The hole was unravelled from a cylinder to plane. Each point represents a radius for a specific position on the periphery of the hole

The maximum diameter of each slice was calculated by identifying two edge points that pass from the $c_{\text {ideal }}$, and the results in Fig. 11a indicate delaminated areas close to the entrance and exit surfaces. The circularity was calculated by the difference between maximum and minimum radii of each slice, with results displayed in Fig. 11b. The hole is digitally represented by 458 slices for the $\mathrm{CD}$ technique and 451 slices for the UAD technique, with each slice having thickness of $14.72 \mu \mathrm{m}$ for CD and $14.76 \mu \mathrm{m}$ for UAD. The represented circles contain numerous defects at the periphery for the entire length of the hole. This method also allows the identification of the exact point that a par-
Fig. 11 Zero millimeter represents the entry plane of the drill and $6.75 \mathrm{~mm}$ the exit plane. a The maximum diameter of each slice of the hole represented throughout the length of the hole. b The circularity of each slice of the hole represented throughout the length of the hole. $\mathbf{c}$ The positioning of each slice represented throughout the length of the hole. d The delaminated area at the exit of the hole represented from 6.2 to $6.75 \mathrm{~mm}$ and represents only the delaminated areas connected to the hole

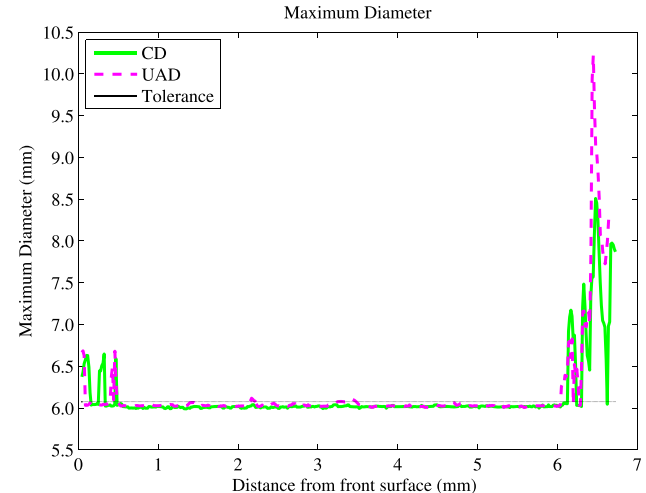

(a)

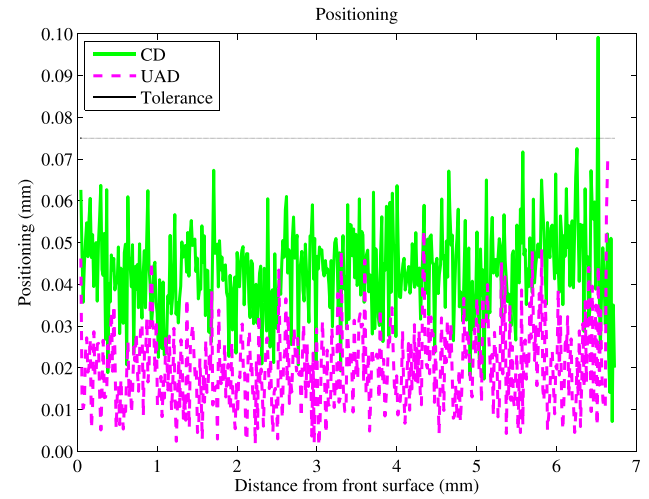

(c)

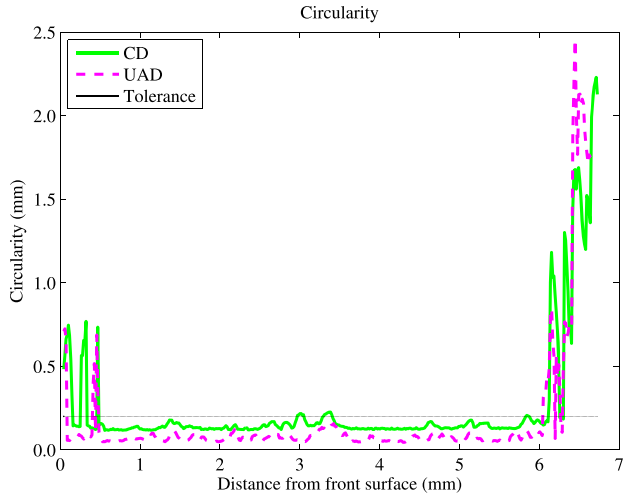

(b)

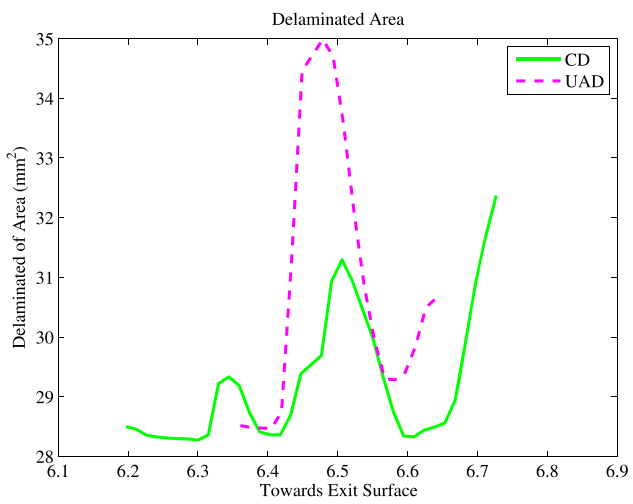

(d) 


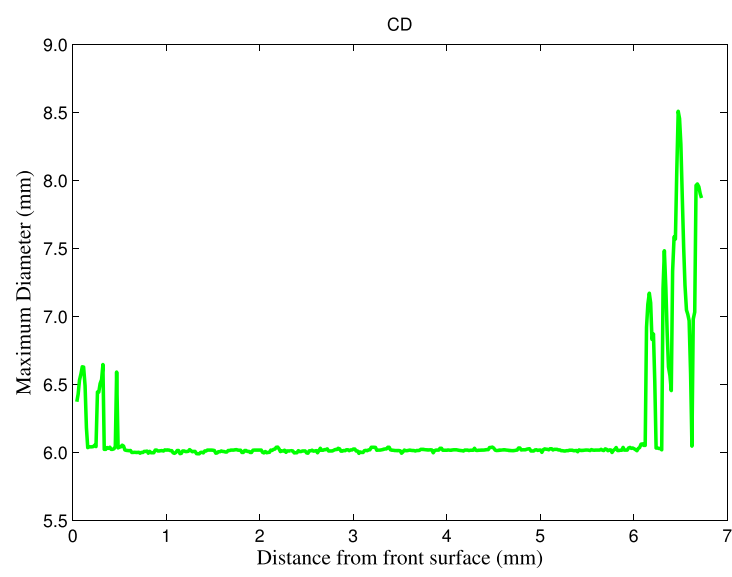

(a)

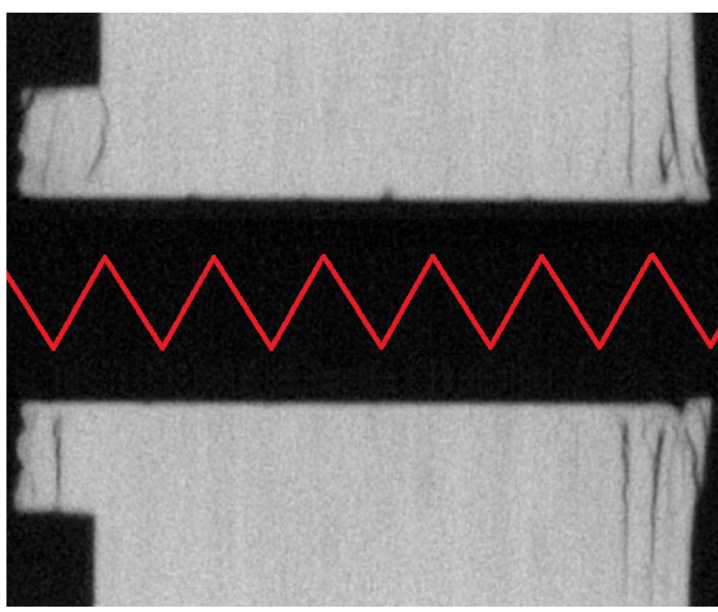

(c)

Fig. 12 The maximum diameter of the hole can assist to identify the delaminated areas, $\mathbf{a}$ shows the maximum diameter of all of the slices. b Shows the maximum diameters of the main body of the hole. $\mathbf{c}$ Shows the corresponding point of the hole for each maximum diameter from

ticular defect is detected, as well as further examination of the slice. Patterns may be able to be detected this way with more experiments. The circularity of both the UAD and $\mathrm{CD}$ demonstrates severe issues close to the exit surface. Defects less than $1 \mathrm{~mm}$ are detected within $0.5 \mathrm{~mm}$ of the entrance surface for $\mathrm{CD}$, with subsequent defects up to the exit region that is smaller than the tolerance specified in Table 4.

The positioning of the hole was measured by the absolute difference between the ideal centre that was identified by the slots and the actual centre $c_{\text {actual }}$ that was identified by the circular Hough transform at each slice, shown in Fig. 11c. The majority of the slices are in tolerance with the values given in Table 4 with the only exceptions, $0.5 \mathrm{~mm}$ from the exit surface. The positioning results can also demonstrate the vibrations of the drill since this is the only variable affecting the positioning after the selection of the intended centre.

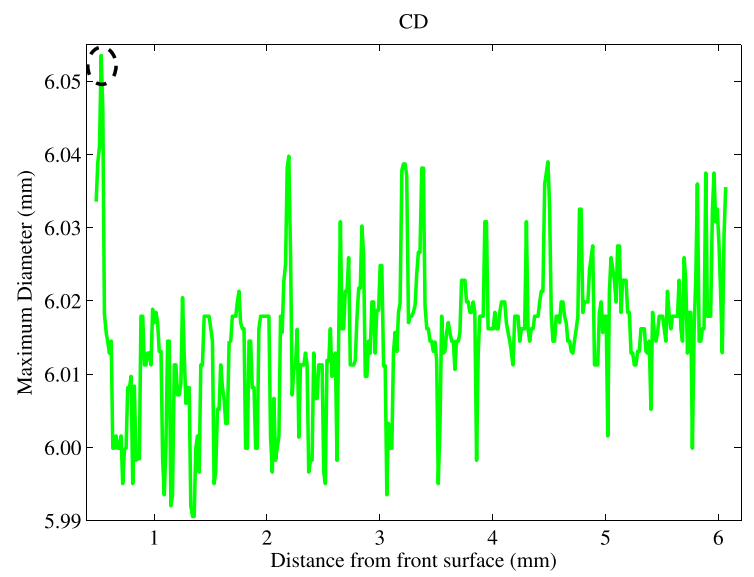

(b)

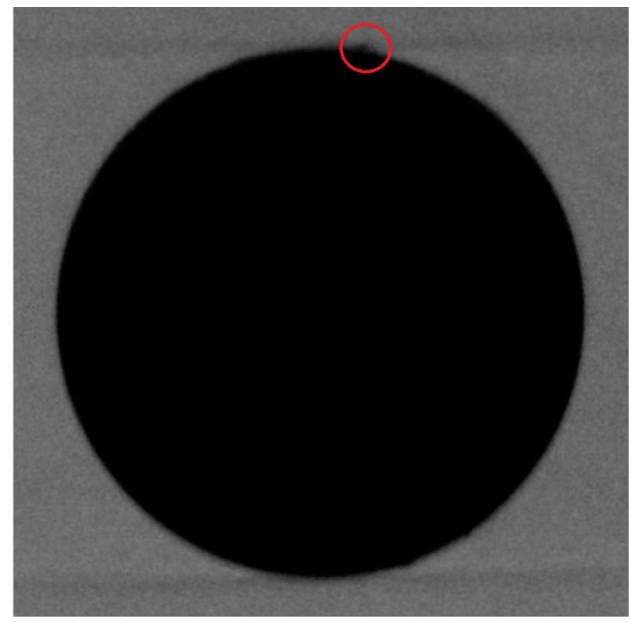

(d)

the graph above. The central hole has been removed in this picture as indicated by the jagged line. d shows the slice of the selected point from (b) and highlights the defect in red

The delaminated area was calculated near the exit by summing up the darker area that exceeds the boundaries of the hole, as shown in Fig. 11d. The results indicate a growth of delaminated area close to the exit surface for both UAD and CT samples.

A more precise examination of $\mathrm{CD}$ hole is provided in Fig. 12. The results representing the maximum diameter are indicating different defects, as well as their size. Figure 12a shows the deviation of maximum diameter from all the slices of the hole, severe exit delamination, seen in Fig. 12c for an example of CD hole, contributes to the sharp peaks observed in this graph. Figure $12 \mathrm{~b}$ concentrates on the maximum diameter of the main body of the hole to better demonstrate the issues that are not related to entrance and exit delamination. A slice is represented in Fig. 12d that represents the slice of the hole at $0.73 \mathrm{~mm}$ from the entrance surface and has a defect marked with red that causes the circled peak in Fig. 12b. 


\section{Conclusions}

This paper presents a novel method of providing dimensional measurements of entire holes machined in CFRPs. It combines computed tomography, measurements from coordinating measuring machine and image processing. It has been developed to provide accurate measurements for different settings and techniques of drilling. The results of this study are used here to demonstrate the potentials of this method, and the assistance that it can provide in manufacturing. This method is the first attempt to study the inner structure of CFRPs.

- CFRP delamination close to entrance and exit surfaces: The results of the analysis show excessive delamination for both UAD and CD close to the entrance and exit surfaces for the cutting speed of $0.0157 \mathrm{~m} / \mathrm{s}$. The maximum diameter results indicate that the main body of the hole can pass the tolerance inspection with the delaminated surface failing for more than $4 \mathrm{~mm}$.

- Out of tolerance circularity: The tolerance of circularity for similar parts in aerospace industry is expected to be $0.1 \mathrm{~mm}$. There are large defects outside this tolerance at the entrance and exit surfaces of the holes, with the defects being more than 0.5 and $2.0 \mathrm{~mm}$ out of tolerance respectively. Both samples would fail inspection.

- Acceptable positioning: The positioning for UAD passes the tolerance inspection and indicates that the technique is adequate while $\mathrm{CD}$ fails the inspection with a crack that reaches $0.1 \mathrm{~mm}$. The positioning results of the CD hole also indicates the misalignment of the drill with radii smaller than $2.95 \mathrm{~mm}$ at $150^{\circ}$ and larger than $3.05 \mathrm{~mm}$ in the horizontally corresponded area, as shown in Fig. 10.

- Problematic areas: Problematic areas were identified through the representation of the entire hole. These areas have a difference up to $0.1 \mathrm{~mm}$ from their surrounding area and indicate the non-homogeneity in the strength of the material. This could also indicate fibre pull-out.

In summary, UAD provides superior circularity, positioning and diameter over CD for the main body of the hole, whereas $\mathrm{CD}$ is better even though out of tolerance, at the entrance and exit surfaces of the holes. Consequently, CD also has a smaller delaminated area.

- CT examination of internal structure and characteristics: The results reliably show the defects and their position with numerous methods of quantification for comparison against given tolerance.

- CT as non-destructive evaluation: Data, which was unreachable in the past with other techniques, can now be achieved using CT.
It is evident that $\mathrm{CT}$ is a suitable non-destructive and noncontact evaluation technique to evaluate machining techniques before they are used in production. A particular advantage is that the calculations were made with limited interactions by the user and consequently the human error is minimised. This demonstrates the ability to use this technology and method for a near automatic quality assessment technique in a mass production environment.

Acknowledgments The authors would like to thank BAE Systems for supporting and EPSRC for funding this project.

\section{References}

1. Adams RD, Cawley P (1988) A review of defect types and nondestructive testing techniques for composites and bonded joints. NDT Int 21:208-222

2. Teti R (2002) Machining of composite materials. CIRP Ann Manuf Technol 51:611-634

3. Pejryd L, Beno T, Carmignato S (2014) Computed tomography as a tool for examining surface integrity in drilled holes in cfrp composites. In: 2nd CIRP Conference on Surface Integrity (CSI), vol 13, pp 43-48

4. Grilo TJ, Paulo RMF, Silva CRM, Davim JP (2013) Experimental delamination analyses of CFRPs using different drill geometries. Compos Part B 45:1344-1350

5. Persson E, Eriksson I, Zackrisson L (1997) Effects of hole machining defects on strength and fatigue life of composite laminates. Compos Part A 28A:141-151

6. Santhanakrishnan G, Krishnamurthy R, Malhotra SK (1988) Machinability characteristics of fibre reinforced plastics composites. J Mech Work Technol 17:195-204

7. Davim JP, Reis P (2003) Study of delamination in drilling carbon fiber reinforced plastics (CFRP) using design experiments. Compos Struct 59:481-487

8. Makhdum F, Norddin DNP, Roy A, Silberschmidt VV (2012) Ultrasonically assisted drilling of carbon fibre reinforced plastics. Solid State Phenom 188:170-175

9. Tsao CC, Hocheng H (2004) Taguchi analysis of delamination associated with various drill bits in drilling of composite material. Int J Mach Tools Manuf 44:1085-1090

10. Abrate S, Walton DA (1992) Machining of composite materials. Part I: Traditional methods. Compos Manuf 3:75-83

11. Hocheng H, Tsao CC (2003) Comprehensive analysis of delamination in drilling of composite materials with various drill bits. $\mathrm{J}$ Mater Process Technol 140:335-339

12. Stone R, Krishnamurthy K (1996) A neural network thrust force controller to minimize delamination during drilling of graphite epoxy laminates. Int J Mach Tools Manuf 36:9851003

13. Gaitonde VN, Karnik SR, Rubio JC, Correia AE, Abrao AM, Davim JP (2008) Analysis of parametric influence on delamination in high speed drilling of carbon fiber reinforced plastic composites. J Mater Process Technol 203:431-438

14. Abrao AM, Faria PE, Rubio JCC, Reis P, Davim JP (2007) Drilling of fiber reinforced plastics: A review. J Mater Process Technol 186:1-7

15. Marques AT, Durao LM, Magalhaes AG, Silva JF, Tavares JMRS (2009) Delamination analysis of carbon fibre reinforced laminates: Evaluation of a special step drill. Compos Sci Technol 69:2376-2382 
16. Anand RS, Patra K, Stiner M (2014) Size effects in micro drilling of carbon fiber reinforced plastic composite. Prod Eng Res Devel 8:301-307

17. Lau WS, Yue TM, Lee TC, Lee WB (1995) Un-conventional machining of composite materials. J Mater Process Technol 48:199-205

18. Piquet R, Ferret B, Lachaud F, Swider P (2000) Experimental analysis of drilling damage in thin carbon/epoxy plate using special drills. Compos Part A 31:1107-1115

19. Park KY, Choi JH, Lee DG (1995) Delamination-free and high efficiency drilling of carbon fiber reinforced plastics. J Compos Mater 29:1988-2002

20. Mohan NS, Ramachanda A, Kulkrni SM (2005) Influence of process paramaters on cutting force and torque during drilling of glass-fiber polyester reinforced composites. Compos Struct 71:407-413

21. Gupta A, Barnes S, McEwen I, Kourra N, Williams MA (2014) Study of cutting speed variation in the ultrasonic assisted drilling of carbon fibre composites. In: Proceeding of the ASME international mechanical engineering congress \& exposition, IMECE, vol 14

22. Goeje MP, Wapenaar KED (1992) Non-destructive inspection of carbon fibre-reinforced plastics using eddy current methods. Composites 23:147-157

23. Pye CJ, Adams RD (1981) Detection of damage in fibre reinforced plastics using thermal field generated during resonant vibration. NDT Int 14:111-118

24. Mook G, Lange R, Koeser O (2001) Non-destructive characterisation of carbon-fibre-reinforced plastics by means of eddy-currents. Compos Sci Technol 61:865-873

25. Mouritz AP, Townsend C, Shah Khan MZ (2000) Non-destructive detection of fatigue damage in thick composites by pulse-echo ultrasonics. Compos Sci Technol 60:23-32

26. Steinberger R, Valagas Leitao TI, Ladstatter E, Pinter G, Billinger W, Lang RW (2006) Infrared thermographic techniques for nondestructive damage characterization of carbon fibre reinforced polymers during tensile fatigue testing. Int J Fatigue 28:13401347

27. Davim JP, Rubio JC, Abrao AM (2007) A novel approach based on digital image analysis to evaluate the delamination factor after drilling composite laminates. Compos Sci Technol 67:1939-1945

28. Seif MA, Khashaba UA, Rojas-Oviedo R (2007) Measuring delamination in carbon/epoxy composites using a shadow moire laser based imaging technique. Compos Struct 79:113-118

29. Raisutis R, Kazys R, Zukauskas E, Mazeika L, Vladisauskas A (2010) Application of ultrasonic guided waves for non-destructive testing of defective CFRP rods with multiple delaminations. NDT E Int 43:416-424

30. Herman GT (2009) Fundamentals of computed tomography image reconstruction from projections. Springer, London
31. Hsieh J (2009) Computed tomography -principles, design, artifacts, and recent advances, 2nd edn. SPIE Press, Washington

32. Kruth JP, Bartscher M, Carmignato S, Schmitt R, De ChiffreL, Weckenmann A (2011) Computed tomography for dimensional metrology. CIRP Ann Manuf Technol 60:821-842

33. Sun W, Brown SB, Leach RK (2012) An overview of industrial $\mathrm{X}$-ray computed tomography. Queen's Printer and Controller of HMSO

34. Welkenhuyzen F, Kiekens K, Pierlet M, Dewulf W, Bleys P, Kruth JP, Voet A (2009) Industrial Computer Tomography for dimensional metrology: Overview of influence factors and improvement strategies. In: OPTIMESS conference

35. Kumar J, Attridge A, Wood PKC, Williams MA (2011) Analysis of the effect of cone-beam geometry and test object configuaration on the measurement accuracy of a computed tomography scanner used for dimensional measurement. Meas Sci Technol 22:3510535120

36. Hiller J, Maisl M, Reindl LM (2012) Physical characterization and performance evaluation of an X-ray micro-computed tomography system for dimensional metrology applications. Meas Sci Technol 23:85404-85422

37. Weib D, Lonardoni R, Deffner A, Kuhn C (2012) Geometric image distortion in flat-panel X-ray detectos and its influence on the accuracy of CT-based dimensional measurements. In: iCT conference

38. Dewulf W, Kiekens K, Tan Y, Welkenhuyzen F, Kruth JP (2013) Uncertainty determination and quantification for dimensional measurements with industrial computed tomography. CIRP Ann Manuf Technol 62:535-538

39. Computed tomography in dimensional measurements. Measurement procedure and comparability. VDI/VDE 2630 Part 1.4 (2010)

40. Lifton JJ, Malcolm AA, McBride JW, Cross KJ (2013) The application of voxel size correction in X-ray Computed Tomography for dimensional metrology. In: Singapore international NDT conference \& exhibition

41. Sivasankar S, Jeyapaul R, Kolappan S, Shaadil NM (2012) Procedural study for roughness, roundness and waviness measurement of EDM drilled holes using image processing technology. Comput Model Inf Process 16:49-63

42. Kourra N, Williams MA, Attridge A, Warnett J, Barnes S, Gupta A (2014) Analysis of drilled holes on carbon fibre material with $\mathrm{X}$-ray computed tomography. In: IEEE international workshop on metrology for aerospace

43. Silva D, Teixera JP, Machado CM (2014) Methodology analysis for evaluation of drilling-induced damage in composites. Int $\mathrm{J}$ Manuf Technol 71:1919-1928

44. Krulikowski A. (1997) Form controls, fundamentals of geometric dimensioning \& tolerancing. Effective Training Inc., Wayne, pp 71-110 